\title{
Tinnitus and Otosclerosis: An Exploratory Study about the Prevalence, Features and Impact in Daily Life
}

\author{
Antonio Fontes Lima1® \\ Fernando Mar $^{1}{ }^{10}$ Luis Dias ${ }^{10}$ \\ ${ }^{1}$ Otorhinolaryngology Service, Hospital de Braga-, Braga, Portugal \\ Int Arch Otorhinolaryngol 2022;26(3):e390-e395.
}

\author{
Isabel Esteves Costa1® Catia Azevedo ${ }^{1 \odot}$
}

\begin{abstract}
Address for correspondence Antonio Fontes Lima, Serviço de Otorrinilaringologia, Hospital de Braga, R. das Comunidades Lusíadas, 133, Braga, Portugal (e-mail: antoniofonteslima24@gmail.com; antonio.lima@hb.min-saude.pt).
\end{abstract}

\begin{abstract}
Introduction Tinnitus is experienced by a significant part of the patients suffering from otosclerosis.

Objective To assess the prevalence of tinnitus in otosclerosis, its main features, and the impact on the daily life.

Methods Patients diagnosed with otosclerosis in 2019 in a tertiary hospital were enrolled in the study. Demographic data were retrieved and, besides a regular audiometric evaluation, the patients underwent acuphenometry to assess the psychoacoustic measurements (pitch and loudness), and the Tinnitus Handicap Inventory (THI).

Results In total, 66 patients fulfilled the inclusion criteria, with a female predominance $(63.6 \% ; n=42)$, and a mean age of 48.7 years. The mean air-bone gap was of $26.3 \mathrm{~dB}$. A total of $72.7 \%$ complained of tinnitus; it was mostly unilateral, identified in the low frequencies, namely $500 \mathrm{~Hz}$, with median loudness of $7.5 \mathrm{~dB}$. The median score on the THI score was of 37 ; most patients had a mild handicap $(33.3 \%, n=16)$, followed by those with a severe handicap $(22.9 \% ; n=11)$. The female gender had a statistically significant association with the presence of tinnitus. The THI scores were higher in middle-aged patients (age groups: 40 to 49 and 50 to 59 years), which was statistically significant. No correlation was found between audiometry results and the prevalence of tinnitus or score on the THI. On the other hand, high-pitched tinnitus, compared to low pitched-tinnitus, was associated with larger air-bone gaps.

Keywords

- tinnitus

- otosclerosis

- psychoacoustic measurements

Conclusion The prevalence of tinnitus in our population was in line with the prevalences reported in the literature. It caused a catastrophic handicap in $22.9 \%$ of the patients. High-pitched tinnitus was associated with higher handicap. Nonetheless, the existence of tinnitus and its severity were not associated with the degree of hearing loss.
\end{abstract}

received

September 13, 2020 accepted after revision

December 7, 2020

published online

November 3, 2021
DOI https://doi.org/ 10.1055/s-0041-1739967. ISSN 1809-9777.

\footnotetext{
(c) 2021. Fundação Otorrinolaringologia. All rights reserved. This is an open access article published by Thieme under the terms of the Creative Commons Attribution-NonDerivative-NonCommercial-License, permitting copying and reproduction so long as the original work is given appropriate credit. Contents may not be used for commercial purposes, or adapted, remixed, transformed or built upon. (https://creativecommons.org/ licenses/by-nc-nd/4.0/) Thieme Revinter Publicações Ltda., Rua do Matoso 170, Rio de Janeiro, RJ, CEP 20270-135, Brazil
} 


\section{Introduction}

Otosclerosis is a disease of the otic capsule and footplate of the stapes in which several changes happen that lead to bony remodeling. ${ }^{1}$

Clinically, this disorder usually presents with hearing loss (HL) complaints, and the audiometry usually shows conductive HL, but sensorineural or mixed HL may occur. However, other clinical signs and symptoms may be associated with the disease, such as tinnitus. Besides being common, tinnitus may be present before hearing loss, and might be the patient's primary complaint. $^{2}$

The reported prevalence of tinnitus in patients suffering from otosclerosis varies between $65 \%$ and $91.6 \%{ }^{3-12}$ The pathophysiology of this symptom is still obscure, but there are some possible explanations: from toxic metabolites derived from the otosclerotic process to altered blood flow to the pathological vascularization, nerve irritation resulting from bone remodeling, and reduction in vibration of the inner-ear fluid. ${ }^{9}$

The definition of tinnitus implies the perception of a sound originated in the ear without an actual external source of sound stimulus. ${ }^{13}$ It is a symptom that can be present in a myriad of otologic and non-otologic pathologies. The former include presbycusis, chronic otitis media, arteriovenous malformations, middle-ear paragangliomas, otosclerosis, amongst others; and the latter include metabolic, cardiovascular, psychiatric, neurological, as well as temporomandibular disorders. Its incidence increases in older patients, and with hearing loss.

Tinnitus is usually described by the following characteristics: unilateral or bilateral; pulsatile or nonpulsatile; persistent or intermittent; and by psychoacoustic measurements. Regarding the latter, there is still a lot of controversy on the subject, but tinnitus is usually classified by its pitch and loudness. Acuphenometry is an exam that aims to find the frequency and intensity that match the pitch and loudness of the tinnitus respectively. ${ }^{13,14}$ Nonetheless, it is not widely used in the audiology routine given the high variability of the tinnitus itself: some patients have two or more tinnitus sounds, and it is not always easy for the patient to establish the predominant one; it can vary over the time in terms of pitch and loudness; low-pitched tinnitus is easily masked by daily sounds, which makes pitch matching difficult. ${ }^{14}$

On the other hand, tinnitus with the same features may generate different feelings and sensations in different patients, and the assessesment of its subjective impact is also an important evaluation. Understanding the impact of this symptom in the patient's daily life is crucial. There are a few validated patient-reported outcome measures (PROMs) regarding this symptom. One of the most widely used is the Tinnitus Handicap Inventory (THI), which has been translated and validated to Portuguese. ${ }^{15,16}$ This instrument is divided into 25 questions, with the items grouped in 3 subscales, namely the functional (THI-F), emotional (THI-E), and catastrophic (THI-C) subscales. A maximum of 100 points may be scored on the THI, and higher scores represent higher impairment in daily life. A grading system was proposed by McCombe et al. ${ }^{17}$ (2001) and recommended for research purposes: a score between 0 and 16 indicates a slight handicap; from 18 to 36, mild; between 38 and 56, moderate; from 58 to 76, severe; and btween 78 and 100 , a catastrophic handicap. The scores on the THI-F vary between 0 and 48; on the THI-E, between 0 and 32; and, on the THI-C, between 0 and 20 .

\section{Objective}

The purpose of the present study is to evaluate the prevalence and features of tinnitus in patients suffering from otosclerosis, through acuphenometry, its association with hearing thresholds and other individual characteristics, and to verify its impact in daily life and its severity through a validated PROM, namely, the THI.

\section{Material and Methods}

After obtaining approval from the institutional Ethical Committee (Reference 96/2020), the patients who were diagnosed with otosclerosis, and with an indication for stapes surgery between the January 1st and December 31st, 2019, were included and prospectively enrolled in the present study. The exclusion criteria were the following: age $<18$ years; previous otologic surgery (including stapes surgery); computed tomography findings suggesting another middle/inner-ear disorder; and incapability to complete the whole assessment.

The following data were obtained: demographics (age and gender); pure-tone audiometry (PTA, which included air conduction $[\mathrm{AC}]$ and bone conduction $[\mathrm{BC}]$ in the frequencies of $250 \mathrm{~Hz}, 500 \mathrm{~Hz}, 1,000 \mathrm{~Hz}, 2,000 \mathrm{~Hz}, 4,000 \mathrm{~Hz}$, and $8000 \mathrm{~Hz}$ ), and air-bone ( $\mathrm{AB}$ ) gap; presence or absence of tinnitus and its features (unilateral or bilateral, persistent or intermittent, and psychoacoustic measurements, namely pitch and loudness); the total score on the THI; and the individual scores on the subscales THI-F, THI-E, and THI-C.

The psychoacoustic measurements were evaluated through acuphenometry, in which matching of the pitch and loudness the patient's tinnitus was performed. Clear information was provided to the patient, namely an explaination that the sound should match in the most accurate way the usual tinnitus after different sounds were offered to the patient. Whenever the tinnitus was unilateral, the sound was offered to the contralateral ear; if the tinnitus was bilateral, the sound was offered to the ear with the best hearing.

Regarding the audiometric findings, the pure-tone average was calculated using the mean hearing level in the frequencies of $500 \mathrm{~Hz}, 1,000 \mathrm{~Hz}, 2,000 \mathrm{~Hz}$, and $4,000 \mathrm{~Hz}$. Concerning the psychoacoustic measurements, the pitch of the tinnitus was further classified as low $(<1,000 \mathrm{~Hz})$ or high $(>2,000 \mathrm{~Hz})$.

\section{Statistical Analysis}

The statistical analysis was performed using the Statistical Package for the Social Sciences (SPSS, IBM Corp., Armonk, NY, US) software, version 23.0 for macOS. Values of $p<0.05$ were used to reject the null hypothesis.

The continuous variables with normal distribution were described as means and standard deviations (SDs); and those without normal distribution were described as medians and interquartile ranges (IQRs). 
Table 1 Demographics of the study sample

\begin{tabular}{|l|l|l|l|}
\hline & $\begin{array}{l}\text { Total } \\
\text { population }\end{array}$ & Male & Female \\
\hline Gender $-\%(n)$ & - & $\begin{array}{l}36.4 \% \\
(\mathrm{n}=24)\end{array}$ & $\begin{array}{l}63.6 \% \\
(\mathrm{n}=42)\end{array}$ \\
\hline $\begin{array}{l}\text { Age (years; mean } \pm \\
\text { standard deviation) }\end{array}$ & $48.7 \pm 9.1$ & $50.7 \pm 8.8$ & $47.6 \pm 9.2$ \\
\hline
\end{tabular}

The Chi-squared $\left(X^{2}\right)$ test was used to evaluate the correlation between two categorical variables. To compare means, the $t$-test was performed; the non-parametric MannWhitney U test was used to compare medians. The Kruskal-Wallis $\mathrm{H}$ test was used to compare medians between more than two independent samples. The Pearson correlation coefficient ( $r$ ) was used to assess the correlation between continuous variables with normal distribution, while the Spearman correlation coefficient $\left(r_{s}\right)$ was used for continuous variables without normal distribution.

\section{Results}

\section{Demographic Features}

A total of 66 patients fulfilled the inclusion criteria (- Table 1 ). There was a female predominance $(63.6 \% ; n=42)$. The mean age of the patients was $48.7 \pm 9.1$ years (range: 35 to 66 years). There was no statistically significant difference in mean age between male and female patients (male: $50.7 \pm 8.8$ years; female: $47.6 \pm 9.2$ years: $\mathrm{t}(64)=1.314 ; p=0.194$ ).

\section{Audiometric Thresholds}

An audiometric evaluation of these patients showed a PTA of $23.1 \pm 8.2 \mathrm{~dB}$ for $\mathrm{BC}, 49.4 \pm 12.1 \mathrm{~dB}$ for $\mathrm{AC}$, and a mean $\mathrm{AB}$ gap of $26.3 \pm 8.6 \mathrm{~dB}$ (-Fig. 1).

\section{Mean audiometry}

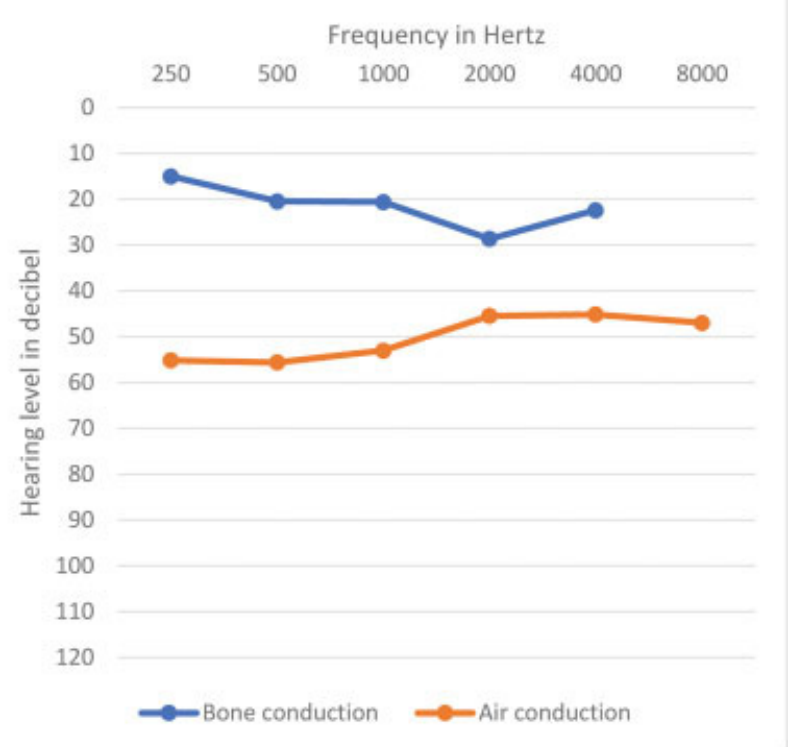

Fig. 1 Mean results of the audiometry.
We did not verify a statistically significant difference in gender regarding the $\mathrm{PTA}$ for $\mathrm{BC}, \mathrm{AC}$, and the $\mathrm{AB}$ gap: $\mathrm{t}$ $(63.9)=1.297, p=0.199 ; \mathrm{t}(64)=1.553, p=0.125$; and $\mathrm{t}$ $(64)=1.072, p=0.288$ respectively.

We verified a correlation between age and the PTA for BC and $\mathrm{AC}$ ( $\mathrm{r}[66]=0.334, p=0.006$; and $\mathrm{r}[66]=0.347$, $p=0.004$ respectively), which means higher thresholds with increasing age. Nonetheless, we did not find a statistically significant correlation between age and the AB gap ( $r$ $[66]=0.168, p=0.179$ ).

\section{Tinnitus Features}

Tinitus was a complaint of $72.7 \%(n=48)$ of the patients. Most of them had unilateral tinnitus $(83.3 \%, \mathrm{n}=40)$, while only $16.7 \%(n=8)$ reported bilateral tinnitus.

Regarding the psychoacoustic measurements (-Table 2 ), most patients $(62.5 \% ; \mathrm{n}=30)$ identified their tinnitus in the low frequencies, mainly $500 \mathrm{~Hz}(37.5 \% ; \mathrm{n}=18)$. The median for loudness was of $7.5 \mathrm{~dB}(\mathrm{IQR}=5)$.

We found a statistically significant association between gender and the existence of tinnitus complaints ( $X^{2}[1$, $\mathrm{n}=66]=9.821 ; p=0.002$ ), with a higher prevalence among the female gender ( $86 \%$ versus $50 \%$ among the male patients). We did not find an association between gender and laterality or pitch of the tinnitus ( $p=1.000$ and $p=0.302$ respectively).

A statistically significant difference in the age of the patients with and without tinnitus complaints was found, with a mean age of $51 \pm 10.6$ years in the former and $45 \pm 4.9$ years in the latter $(\mathrm{t}[61]=-3.138 ; p=0.003)$. No difference was found regarding the mean age between patients with high- or low-pitched tinnitus ( $\mathrm{t}[46]=-0.336 ; p=0.739$ ).

We did not verify a statistically significant difference in PTA for BC, AC, and the AB gap between patients who complained and did not complain of tinnitus (t-test: $p=0.544, p=0.862$, and $p=0.885$ respectively). On the other

Table 2 Presence of tinnitus and features evaluated through acuphenometry (subjective pitch and subjective loudness)

\begin{tabular}{|l|l|l|}
\hline & $\%$ & $\mathbf{n}$ \\
\hline Patients with tinnitus complaints & $72.7 \%$ & 48 \\
\hline Subjective pitch & & \\
\hline $125 \mathrm{~Hz}$ & $4.2 \%$ & 2 \\
\hline $250 \mathrm{~Hz}$ & $12.5 \%$ & 6 \\
\hline $500 \mathrm{~Hz}$ & $37.5 \%$ & 18 \\
\hline $1,000 \mathrm{~Hz}$ & $8.3 \%$ & 4 \\
\hline $2,000 \mathrm{~Hz}$ & $18.8 \%$ & 9 \\
\hline $4,000 \mathrm{~Hz}$ & $14.6 \%$ & 7 \\
\hline $6,000 \mathrm{~Hz}$ & $4.2 \%$ & 2 \\
\hline Subjective loudness & & \\
\hline $5 \mathrm{~dB}$ & $50 \%$ & 24 \\
\hline $10 \mathrm{~dB}$ & $29.2 \%$ & 14 \\
\hline $15 \mathrm{~dB}$ & $20.8 \%$ & 10 \\
\hline Patients with no tinnitus complaints & $27.3 \%$ & 16 \\
\hline
\end{tabular}


Table 3 Scores on the Tinnitus Handicap Inventory (THI) and its subscales

\begin{tabular}{|l|l|l|}
\hline & Median & Interquartile range \\
\hline THI & 37 & 45 \\
\hline THI-Functional & 16 & 22 \\
\hline THI-Emotional & 9 & 16 \\
\hline THI-Catastrophic & 8 & 8 \\
\hline
\end{tabular}

hand, regarding the pitch of the tinnitus, a statistically significant difference was found regarding the $A B$ gap, which was higher in high-pitched tinnitus compared to low-pitched tinnitus $(30.2 \pm 9.4 \mathrm{~dB}$ in the former, and $23.7 \pm 7 \mathrm{~dB}$ in the latter; $t[28.275]=-2.561 ; p=0.016)$; no statistically significant diference was found concerning the mean PTA for BC and $A C$ between patients with high- or low-pitched tinnitus.

\section{Severity of the Tinnitus - THI Scores}

The median THI score (-Table $\mathbf{3}$ ) among the patients who complained of tinnitus was of $37(\mathrm{IQR}=45)$. Most patients had a mild handicap (33.3\%; $n=16$ ), followed by a severe handicap (22.9\%; $\mathrm{n}=11$ ) (-Table 4).

Regarding gender (-Table 5), we found a statistically significant difference between male and female patients regarding the THI-C (U test; $p=0.03$ ), with higher scores among male patients. The male patients also scored higher on the THI, THI-F, and THI-E, but this difference was not statistically significant.

We found no correlation between age and tinnitus severity $\left(r_{s}=-0.109, p=0.459\right.$ for the THI; $r_{s}=-0.164, p=0.265$ for the THI-F; $r_{s}=0.031, p=0.834$ for the THI-E; and $r_{s}=0.026$, $p=0.858$ for the THI-C; - Table 6 ). We further subdivided our population according to age groups, and verified that higher scores were found among those aged 40 to 49 years, followed by those aged 50 to 59 year; the difference among age groups was statistically significant for the THI $(H[48]=16.223 ; p=0.001)$, the THI-F (H [48] =10.836; $p=0.013]$, THI-E (H [48] =21.417; $p<0.001)$, and the THI-C $(\mathrm{H}[48]=11.359 ; p=0.010)$.

No correlation was found regarding $B C, A C$, or the $A B$ gap and the scores on the THI, THI-F, THI-E, or THI-C.

When evaluating tinnitus severity according to the psychoacoustic measurements, we found that patients with high-pitched tinnitus scored higher on the THI and all of its subscales, but only statistically significant differences

Table 4 Distribution of the Tinnitus Handicap Inventory through categories

\begin{tabular}{|l|l|l|}
\hline & $\%$ & $\mathbf{n}$ \\
\hline Slight handicap (0-16) & $16.7 \%$ & 8 \\
\hline Mild handicap (18-36) & $33.3 \%$ & 16 \\
\hline Moderate handicap (38-56) & $16.7 \%$ & 8 \\
\hline Severe handicap (58-76) & $22.9 \%$ & 11 \\
\hline Catastrophic handicap (78-100) & $10.4 \%$ & 5 \\
\hline
\end{tabular}

Table 5 Scores on the Tinnitus Handicap Inventory (THI) and its subscales divided by gender

\begin{tabular}{|l|l|l|l|}
\hline & Male & Female & $p$-value \\
\hline THI & $60(\mathrm{IQR}=44)$ & $34(\mathrm{IQR}=34)$ & 0.146 \\
\hline THI-Functional & $29(\mathrm{IQR}=24)$ & $16(\mathrm{IQR}=21)$ & 0.159 \\
\hline THI-Emotional & $18(\mathrm{IQR}=16)$ & $6(\mathrm{IQR}=13)$ & 0.302 \\
\hline THI-Catastrophic & $12(\mathrm{IQR}=6)$ & $6(\mathrm{IQR}=18)$ & 0.030 \\
\hline
\end{tabular}

Abbreviation: IQR, interquartile range.

Note: *Mann-Whitney U test for independent samples.

were only found for the THI, THI-E, and THI-C. Moreover, we found a correlation between the reported pitch of the tinnitus (-Table 7) and the THI, THI-E, and THI-C, which means that higher pitches correlated with higher scores $\left(r_{s}=0.355, p=0.013 ; r_{s}=0.334, p=0.020 ;\right.$ and $r_{s}=0.537$, $p<0.001$ respectively). On the other hand, there was no correlation concerning the scores on the THI, THI-F, THI-E, or $\mathrm{THI}-\mathrm{C}$ and subjective tinnitus loudness.

\section{Discussion}

Despite being a well-known symptom related to otosclerosis, tinnitus has not been widely addressed in the literature. Most of the few studies that analyze this symptom do it on a retrospective basis, relying on clinical records or questionnaires after surgery regarding preoperative symptoms, which might influence the results.

The present study showed a prevalence of $72.7 \%$ of tinnitus in otosclerosis patients. This finding is within the range reported in the the literature. Gristwood and Venables ${ }^{3}$ (2003), in a study on tinnitus in otosclerosis patients with the large sample, reported a slightly lower prevalence (the lowest reported in the literature), of $65 \%$, but their data was based on a retrospective analysis of medical records. On the opposite side, a higher prevalence was reported by Bast et al. ${ }^{9}$ (2013), of 82\% (although this study was based on a questionnaire regarding tinnitus applied after surgery), and Sobrinho et al. $^{11}$ (2004), of $91.6 \%$.

Regarding the features of tinnitus, including the psychoacoustic measurements, among the sample of the present study, tinnitus was most commonly reported on the side of the disease; low-pitched tinnitus was more frequent than high-pitched tinnitus; it was identified with a median loudness of $7.5 \mathrm{~dB}$. On the other hand, Ismi et al. ${ }^{8}$ (2017) and Ayache et al. ${ }^{6}$ (2003) found that high-pitched tinnitus was the most frequent among patients with otosclerosis.

As for the severity of the tinnitus, we found that $48.5 \%$ of the patients with otosclerosis had a moderate to catastrophic handicap. This percentage is slightly higher than the one found in the study by Skarżyński et al. ${ }^{5}$ (2019), of $40 \%$, but significantly lower than the $83 \%$ found by Rajati et al. ${ }^{12}$ (2012).

The data of the present study indicated an association of the prevalence of tinnitus with the female gender. Other authors $^{3,5,18}$ also found higher rates of tinnitus complaints among female patients. Only one study ${ }^{11}$ found the opposite, 
Table 6 Scores on the Tinnitus Handicap Inventory (THI) and its subscales divided by age group

\begin{tabular}{|l|l|l|l|l|l|}
\hline Age group (years) & $\mathbf{n}$ & THI & THI-Functional & THI-Emotional & THI-Catastrophic \\
\hline $30-39$ & 8 & $25(\mathrm{IQR}=25)$ & $14(\mathrm{IQR}=24)$ & $4(\mathrm{IQR}=5)$ & $6(\mathrm{IQR}=2)$ \\
\hline $40-49$ & 19 & $56(\mathrm{IQR}=42)$ & $28(\mathrm{IQR}=18)$ & $20(\mathrm{IQR}=12)$ & $12(\mathrm{IQR}=12)$ \\
\hline $50-59$ & 12 & $35(\mathrm{IQR}=24)$ & $16(\mathrm{IQR}=22)$ & $8(\mathrm{IQR}=12)$ & $12(\mathrm{IQR}=6)$ \\
\hline $60-69$ & 9 & $18(\mathrm{IQR}=53)$ & $8(\mathrm{IQR}=29)$ & $6(\mathrm{IQR}=15)$ & $5(\mathrm{IQR}=6)$ \\
\hline
\end{tabular}

Abbreviation: IQR, interquartile range.

Table 7 Scores on the Tinnitus Handicap Inventory (THI) and its subscales divided by pitch of the tinnitus (low versus high)

\begin{tabular}{|l|l|l|l|}
\hline & $\begin{array}{l}\text { Subjective } \\
\text { low-pitched } \\
\text { tinnitus }\end{array}$ & $\begin{array}{l}\text { Subjective } \\
\text { high-pitched } \\
\text { tinnitus }\end{array}$ & $p$-value* \\
\hline THI & $26(\mathrm{IQR}=34)$ & $62(\mathrm{IQR}=47)$ & 0.030 \\
\hline THI-Functional & $16(\mathrm{IQR}=20)$ & $30(\mathrm{IQR}=24)$ & 0.236 \\
\hline THI-Emotional & $6(\mathrm{IQR}=13)$ & $16(\mathrm{IQR}=15)$ & 0.034 \\
\hline THI-Catastrophic & $6(\mathrm{IQR}=7)$ & $14(\mathrm{IQR}=10)$ & $<0.001$ \\
\hline
\end{tabular}

Abbreviation: IQR, interquartile range.

Note: *Mann-Whitney $\mathrm{U}$ test for independent samples.

namely a male predominance in tinnitus complaints. The literature regarding tinnitus in general (and not specifically in otosclerosis) is also consensual, stating that the female gender has a higher prevalence of subjective tinnitus. ${ }^{3}$ On the other hand, the correlation between the severity of the tinnitus and gender is even more divisive in the literature: despite a statistically significant difference regarding higher scores on the THI-C in male patients in the present study, most authors $5,6,11,12$ did not find any association between gender and the severity of the tinnitus.

Regarding age, we found a statistically significant difference in the mean age between patients with and without tinnitus complaints, with older patients complaining more often of this symptom; on the other hand, we did not find a correlation between age and severity of the tinnitus, although middle-aged patients (those aged 40 to 49 and 50 to 59 years) scored higher on the THI, which was statistically significant. Skarżyński et al. ${ }^{5}$ also found that the prevalence and severity of the tinnitus increase with age among female patients, but not among male patients. ${ }^{5}$ Other studies ${ }^{3}$ did not find any significant difference. We then may hypothesize that, although older patients with otosclerosis complain more of tinnitus, middle-aged patients have more difficulty coping with this symptom; therefore, its subjective severity is worse. On the other hand, younger and older patients do not value this symptom so much.

Our results showed no statistically significant correlation between the degree of hearing loss (PTA for $B C, A C$, or the $A B$ gap) and the existence of tinnitus, nor with the severity of tinnitus evaluated through the THI. Axelsson and Ringdahl ${ }^{19}$ (1989) reported higher rates of tinnitus complaints among patients with different types and causes of hearing loss compared to patients without hearing loss, but failed to prove the existence of a correlation between the different degrees of hearing loss and tinnitus severity. On the contrary, more recently, Moon et al. ${ }^{20}$ (2018) found that severe hearing loss was a predictive factor for higher impairment in quality of life regarding tinnitus. Concerning only patients with otosclerosis, the literature is also controversial: Gristwood and Venables ${ }^{3}$ (2003), and Skarżyński et al. $^{5}$ (2019) found that the prevalence of tinnitus complaints initially increased with increasing $A B$ gap, but fell when the $A B$ gap became larger, with no correlation to tinnitus severity; on the other hand, authors such as Rajati et al. ${ }^{12}$ (2012), Sobrinho et al. ${ }^{11}$ (2004), and Ayache et al. ${ }^{6}$ (2003) found no correlation between preoperative hearing levels and tinnitus complaints and severity.

There was no statistically significant association regarding the psychoacoustic measurements and gender or age. Despite the absence of correlation between audiometry results and the existence of tinnitus, our data indicate that the psychoacoustic measurements of tinnitus in patients with otosclerosis correlate with its severity: those with high-pitched tinnitus have significantly higher scores on the THI, THI-E, and THI-C; moreover, there is a positive correlation between pitch and THI scores, with higher pitches usually associated with higher scores on the THI. High-pitched tinnitus also was associated with a higher $A B$ gap: this means that more severe otosclerosis is associated with this type of pitch, which, in turn, is associated with higher impairment related to the associated tinnitus. Contrarily, subjective loudness did not correlate with the THI scores. Flores et al. ${ }^{21}$ (2016), who evaluated patients with noise-induced hearing loss, did not find any correlation between the pitch of the tinnitus and audiometric results, but found an inverse correlation between loudness and audiometric results (the greater the hearing loss, the lower the loudness). Psychoacoustic measurements have not been widely applied in patients suffering from otosclerosis, which makes other comparisons impossible.

\section{Advantages and Limitations of the Study}

The present study was performed with a standardized evaluation protocol, in which the patients were prospectively enrolled, which makes our results more accurate than those of some of the previously-published studies. On the other hand, the present study included only patients diagnosed with otosclerosis who had a surgical indication, excluding patients with mild forms of the disease and those who did not want to undergo surgery. 
Although tinnitus is a subjective symptom, its evaluation is difficult and controversial. It can present in numerous forms, and can vary throughout time in the same patient. Measuring tinnitus through pitch and loudness, therefore, can be an overly-simplified way to assess its true characteristics, although the most frequently used. The fact that we chose an approved and widely used PROM, the THI, to evaluate the impact of the symptom in daily life is also an advantage, by making future comparisons with studies regarding otosclerosis and other causes of hearing loss and tinnitus easier.

Finally, the present study only addresses the preoperative complaints of tinnitus; although surgery is associated with improvement of the tinnitus, it is still controversial which factors influence the resolution of the symptom., ${ }^{4,22,23}$ Regarding the subject, these same patients were prospectively enrolled in a follow-up protocol to complete the second phase of the study: to evaluate the existence and severity of the tinnitus, the relationship with the improvement in hearing after the surgical approach, and the impact of the different techniques and prostheses (stapedotomy versus stapedectomy; Teflon versus titanium prostheses, as well as length and diameter).

\section{Conclusions}

In our population suffering from otosclerosis, tinnitus had a prevalence of $73.7 \%$, with a significantly higher prevalence in the female gender and older patients. This symptom was the source of a moderate to catastrophic impairment in daily life in $50 \%$ of these patients. Despite the fact that most of the patients identified their tinnitus in the low frequencies, high-pitched tinnitus correlated with a higher handicap in daily life. No statistically significant correlation was found between tinnitus and the degree of hearing loss.

Conflict of Interests

The authors have no conflict of interests to declare.

\section{References}

1 Rudic M, Keogh I, Wagner R, et al. The pathophysiology of otosclerosis: Review of current research. Hear Res 2015;330(Pt A):51-56. Doi: 10.1016/j.heares.2015.07.014

2 Lima AdaS, Sanchez TG, Marcondes R, Bento RF. The effect of stapedotomy on tinnitus in patients with otospongiosis. Ear Nose Throat J 2005; 84(07):412-414. Doi: 10.1177/014556130508400710

3 Gristwood RE, Venables WN. Otosclerosis and chronic tinnitus. Ann Otol Rhinol Laryngol 2003;112(05):398-403. Doi: $10.1177 / 000348940311200502$

4 Skarżyński H, Gos E, Dziendziel B, Raj-Koziak D, Włodarczyk EA, Skarżyński PH. Clinically important change in tinnitus sensation after stapedotomy. Health Qual Life Outcomes 2018;16(01):208. Doi: 10.1186/s12955-018-1037-1

5 Skarżyński PH, Dziendziel B, Gos E, et al. Prevalence and severity of tinnitus in otosclerosis: Preliminary findings from validated questionnaires. J Int Adv Otol 2019;15(02):277-282. Doi: 10.5152/iao.2019.5512
6 Ayache D, Earally F, Elbaz P. Characteristics and postoperative course of tinnitus in otosclerosis. Otol Neurotol 2003;24(01): 48-51. Doi: 10.1097/00129492-200301000-00011

7 Glasgold A, Altmann F. The effect of stapes surgery on tinnitus in otosclerosis. Laryngoscope 1966;76(09):1524-1532. Doi: 10.1002/ lary. 5540760904

8 Ismi O, Erdogan O, Yesilova M, Ozcan C, Ovla D, Gorur K. A estapedotomia melhora o zumbido em pacientes com otosclerose? Rev Bras Otorrinolaringol (Engl Ed) 2017;83(05):568-573. Doi: 10.1016/j.bjorl.2016.07.001

9 Bast F, Mazurek B, Schrom T. Effect of stapedotomy on preoperative tinnitus and its psychosomatic burden. Auris Nasus Larynx 2013;40(06):530-533. Doi: 10.1016/j.anl.2013.04.006

10 Szymański M, Gołabek W, Mills R. Effect of stapedectomy on subjective tinnitus. J Laryngol Otol 2003;117(04):261-264. Doi: 10.1258/00222150360600841

11 Sobrinho PG, Oliveira CA, Venosa AR. Long-term follow-up of tinnitus in patients with otosclerosis after stapes surgery. Int Tinnitus J 2004; 10(02):197-201http://www.ncbi.nlm.nih.gov/pubmed/15732523

12 Rajati M, Poursadegh M, Bakhshaee M, Abbasi A, Shahabi A. Outcome of stapes surgery for tinnitus recovery in otosclerosis. Int Tinnitus J 2012;17(01):42-46. Doi: 23906826

13 Seimetz BMA, Teixeira AR, Rosito LPS, Flores LS, Pappen $\mathrm{CH}$, Dall'igna C. Pitch and loudness tinnitus in individuals with presbycusis. Int Arch Otorhinolaryngol 2016;20(04):321-326. Doi: 10.1055/s-0035-1570311

14 Henry JA, Meikle MB. Psychoacoustic measures of tinnitus. J Am Acad Audiol 2000;11(03):138-155

15 Newman CW, Jacobson GP, Spitzer JB. Development of the Tinnitus Handicap Inventory. Arch Otolaryngol Head Neck Surg 1996; 122(02):143-148. Doi: 10.1001/archotol.1996.01890140029007

16 de Oliveira V, Meneses RF. Balanço da utilização da versão portuguesa do Tinnitus Handicap Inventory (THI). 2007

17 McCombe A, Baguley D, Coles R, McKenna L, McKinney C, WindleTaylor PBritish Association of Otolaryngologists, Head and Neck Surgeons. Guidelines for the grading of tinnitus severity: the results of a working group commissioned by the British Association of Otolaryngologists, Head and Neck Surgeons, 1999. Clin Otolaryngol Allied Sci 2001;26(05):388-393. Doi: 10.1046/ j.1365-2273.2001.00490.x

18 Gersdorff M, Nouwen J, Gilain C, Decat M, Betsch C. Tinnitus and otosclerosis. Eur Arch Otorhinolaryngol 2000;257(06):314-316. Doi: $10.1007 / \mathrm{s} 004059900138$

19 Axelsson A, Ringdahl A. Tinnitus-a study of its prevalence and characteristics. $\mathrm{Br} \mathrm{J}$ Audiol 1989;23(01):53-62. Doi: $10.3109 / 03005368909077819$

20 Moon KR, Park S, Jung Y, Lee A, Lee JH. Effects of anxiety sensitivity and hearing loss on tinnitus symptom severity. Psychiatry Investig 2018;15(01):34-40. Doi: 10.4306/pi.2018.15.1.34

21 Flores LS, Teixeira AR, Rosito LP, Seimetz BM, Dall'Igna C. Pitch and Loudness from Tinnitus in Individuals with Noise-induced Hearing Loss. Int Arch Otorhinolaryngol 2016;20(03):248-253. Doi: 10.1055/s-0035-1562935

22 Dziendziel B, Skarżyński H, Gos E, Skarżyński PH. Tinnitus Severity Change Following Stapedotomy in Patients With Otosclerosis. Otol Neurotol 2019;40(05):578-583. Doi: 10.1097/MAO.000 0000000002240

23 Dziendziel B, Skarzynski H, Gos E, Skarzynski PH. Changes in Hearing Threshold and Tinnitus Severity after Stapes Surgery: Which Is More Important to the Patient's Quality of Life? ORL J Otorhinolaryngol Relat Spec 2019;81(04):224-233. Doi: 10.1159/ 000500992 